\title{
TÉCNICA DE EXPOSIÇÃO DA JUNÇÃO ESOFAGOGÁSTRICA OBTIDA POR MEIO DE AFASTADOR FLEXÍVEL DE FÍGADO EM CIRURGIA BARIÁTRICA: ENSAIO CLÍNICO RANDOMIZADO
}

\author{
TECHNIQUE OF EXPOSURE OF THE ESOPHAGOGASTRIC JUNCTION OBTAINED BY THE FLEXIBLE LIVER \\ RETRACTOR IN BARIATRIC SURGERY: A RANDOMIZED CONTROLLED TRIAL
}

Rodrigo Feitosa de Albuquerque Lima BABADOPULOS ${ }^{1}{ }^{\circ}$, Luiz Gonzaga de MOURA-JR ${ }^{1}{ }^{\oplus}$, Vagnaldo FECHINE $^{2}$, Marina Becker Sales ROCHA ${ }^{2 \oplus}$, Natalícia ANTUNES ${ }^{3}{ }^{\circ}$, Thomaz Alexandre COSTA $^{2 \oplus}$, Bruno Almeida COSTA $^{2 \oplus}$, Manoel Odorico DE-MORAES ${ }^{2}$

RESUMO - RACIONAL: Os afastadores clássicos de cirurgia laparoscópica são geralmente rígidos, necessitando de uma incisão adicional para sua instalação ou de um auxiliar para manuseio durante o ato cirúrgico e ainda, podem envolvem risco de injúria hepática. OBJETIVOS: Avaliar e validar uma técnica de exposição da junção esofagogástrica obtida pelo afastador flexível de fígado em cirurgia bariátrica comparando sua eficácia com a de afastador classicamente utilizado para este fim. MÉTODOS: Tratou-se de um estudo prospectivo, aberto, controlado e comparativo em pacientes com indicação de cirurgia, distribuídos de forma randomizada em dois grupos: clássico (controle) e afastador flexível (teste). RESULTADOS: Foram incluídos 100 pacientes $(n=50$ grupo controle, $n=50$ grupo teste), sem diferença estatística na distribuição por idade e por morbidades, havendo diferença estatística somente no gênero (grupo controle obteve proporção maior de homens, $p=0,020$ ). Em relação ao tempo médio de realização das operações, não foi constatada diferença estatística. No quesito visibilidade, verificou-se que $100 \%$ dos pacientes do grupo afastador flexível obteve nível de visibilidade ótima, porém sem significância estatística com relação ao grupo clássico (94\%). Invariavelmente, foi necessário um portal a mais de trocarte quando do uso do afastador clássico. CONCLUSÃO: O afastador flexível de fígado demonstrou-se seguro, eficaz, ergonômico, de baixo custo, de perfil estético satisfatório, não requerendo instrumental específico para uso ou nova curva de adaptação e aprendizado para manuseio.

DESCRITORES: Cirurgia bariátrica. Junção esofagogástrica. Laparoscopia. Obesidade. Derivação gástrica.

ABSTRACT - BACKGROUND: In the Roux-en-Y gastric bypass technique, classic laparoscopic surgical retractors are usually rigid, require an additional incision for its installation, or must be handled by an assistant during the surgical procedure, involving a risk of liver injury. AIM: The aim of this study was to evaluate and validate a technique of the esophagogastric junction exposure obtained by the flexible liver retractor in bariatric surgery, comparing its efficacy with the retractor classically used for this purpose. METHODS: This study was performed as a randomized, open, prospective, controlled, and comparative design in patients with medical indications of bariatric surgery. The subjects were distributed in the classic (control) and flexible (test) retractor groups. RESULTS: A total of 100 patients $(n=50$ control group, $n=50$ test group) were included. No statistically significant difference was observed in the mean duration of surgery. Regarding visibility, $100 \%$ of the patients in the flexible retractor group demonstrated an optimal visibility level, although without statistical significance concerning the classic retractor group (94\%). Invariably, carrying a trocar was necessary when using the classic retractor. CONCLUSIONS: The flexible liver retractor is safe, effective, ergonomic, and inexpensive. Furthermore, it presented a satisfactory aesthetic profile, and the use of specific instruments, new adaptation curve, and training for its handling were not required.

HEADINGS: Bariatric surgery. Esophagogastric junction. Obesity. Laparoscopy. Gastric bypass.

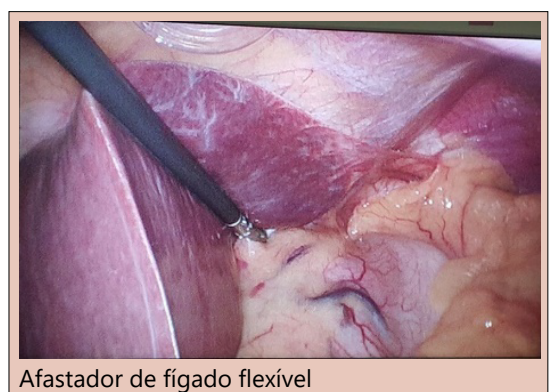

\section{Mensagem central}

O afastador flexível de fígado é seguro, eficaz, ergonômico, de baixo custo e com perfil estético satisfatório para ser utilizado como alternativa aos afastadores atualmente disponíveis.

\begin{tabular}{|l|}
\hline Perspectivas \\
\hline O afastador flexível de fígado proporciona \\
exposição adequada e segura da junção \\
esofagogástrica em procedimentos em que o \\
fígado dificulta a visibilidade, dispensando o \\
uso de instrumentos específicos ou curva de \\
adaptação e treinamento. Também atende às \\
características de um equipamento cirúrgico \\
minimamente invasivo, podendo ser utilizado \\
em operações de portal único, tornando-o \\
adequado para ser utilizado como alternativa aos \\
afastadores atualmente disponíveis.
\end{tabular}

Trabalho realizado na 'Unidade de Cirurgia Bariátrica, Hospital Geral Dr. César Cals, Fortaleza, CE, Brasil; ${ }^{2}$ Centro de Pesquisa e Desenvolvimento de Medicamentos, Unidade de Farmacologia Clínica, Universidade Federal do Ceará, Fortaleza, CE, Brasil; ${ }^{3}$ Departamento de Farmacologia, Faculdade de Ciências Médicas, Universidade Estadual de Campinas - Unicamp, Campinas, São Paulo - SP, Brasil

Como citar esse artigo: Babadopulos RFAL, Moura-Jr LG, Fechine V, Rocha MBS, Antunes N, Costa TA, BA, De-Moraes MO. Técnica de exposição da junção esofagogástrica obtida por meio de afastador flexível de fígado em cirurgia bariátrica: Ensaio clínico randomizado. ABCD Arq Bras Cir Dig. 2021;34(4):e1631. https://doi.org/10.1590/0102-672020210002e1631

Correspondência:

Marina Becker Sales Rocha

Email: marinabecker@hotmail.com.br
Financiamento: Nenhum

Conflito de Interesse: Nenhum

Recebido: 04/11/2020

Aceito: 20/04/2021 


\section{INTRODUÇÃO}

tualmente, a cirurgia bariátrica é aceita como método
efetivo de tratamento para a obesidade mórbida ${ }^{20}$.
Entretanto, estas operações são sempre grandes e complexas, devido ao excesso de peso eas comorbidades associadas. Além disso, podem apresentar morbidade pós-operatória, como fistulas, abcessos, sangramentos, complicações pulmonares e cardiovasculares, que necessitem de um período de recuperação ${ }^{18,21}$.

Do ponto de vista cirúrgico, talvez a complicação mais temida e potencialmente fatal, mesmo que diagnosticada precocemente, seja a fístula da bolsa gástrica no ângulo esofagogástrico (His), região de menor vascularização gástrica ${ }^{3} e$ de maior grau de dificuldade de exposição durante a operação.

Como advento da laparoscopia e o desenvolvimento denovas tecnologias, o sentido do tato tem sido perdido. Entretanto, as imagens e a visualização dos campos melhoraram. Esse fato não é diferente na cirurgia bariátrica, na qual o fígado de pacientes obesos mórbidos pode apresentar lobo esquerdo hipertrofiado e gorduroso, o qual,por sua vez, pode dificultar a visualizaçãoquanto a localização do ângulo esofagogástrico abaixo do lobo esquerdo do fígado, tornando a técnica ainda mais desafiadora para os cirurgiões bariátricos ${ }^{1}$, considerando que a retração do fígado é necessária para obter-se bom campo de visão ${ }^{16}$

A maioria dos cirurgiões experimenta dificuldade em afastar fígado esquerdo hipertrófico e esteatótico ${ }^{12}$. Geralmente, os afastadores "convencionais" de cirurgia laparoscópica são rígidos, necessitam de uma incisão adicional para sua instalação ou de manuseio por um auxiliar durante o ato cirúrgico e envolvem risco de injúria hepática ${ }^{13}$. Além disso, a incisão adicional eleva o risco relativo à ferida e o número de cicatrizes.

Durante $014^{\circ}$ Congresso Mundial da Federação Internacional para a Cirurgia de Obesidade e Transtornos Metabólicos de Cirurgia Bariátrica (IFSO, 2009) em Paris, foi apresentado um método de afastamento hepático, no qual apósa instalação do pneumoperitônio, uma agulha reta era introduzida na cavidade abdominal através de punção em epigástrio. Então, o lobo esquerdo do fígado era inicialmente transfixado de sua superfície parietal para a visceral e, utilizando-se da mesma agulha, transfixado novamente, através de outro ponto de inserção, em sentido contrário (de face visceral para parietal). Posteriormente, a agulha era retirada da cavidade abdominal, também por punção em epigástrio de dentro para fora do abdome. Com isso, o fígado esquerdo era suspenso e assim, a área de trabalho (junção esofagogástrica) exposta adequadamente, sem a necessidade de introdução de um afastador de fígado clássico que ocuparia um trocarte adicional através de nova incisão. Isto eliminaria a necessidade de manipulação do instrumento por auxiliar durante o procedimento ${ }^{8}$. Contudo, havia o risco de sangramento devido a dupla transfixação hepática.

A partir da observação destes métodos explicitados no congresso citado, a equipe de cirurgiões do Núcleo do Obeso do Ceará desenvolveu um novo tipo de afastador hepático (registro de patente em andamento), com o propósito de trazer os mesmos benefícios quanto à exposição da junção esofagogástrica, porém adicionando as seguintes vantagens: supressão de uma incisão na pele, ausência de necessidade de manipulação do instrumento por médico auxiliar e não causar injúria ao fígado ou elevar o risco de sangramento.

Então, desde o ano de2009, este mesmo grupo de cirurgiões tem regularmente utilizado o afastador flexível de fígado em suas operações bariátricas, apresentando o modelo e a técnica de remoção em congressos brasileiros e internacionais ${ }^{10,11}$. Entretanto, apesar do dispositivo já ser utilizado rotineiramente, havia a necessidade da realização de um ensaio clínico para demonstrar, nos moldes científicos, a sua segurança e eficácia, validando assim o afastador flexível de fígado.

Portanto, o objetivo desse estud o foi avaliar a segurança, eficácia e validar o afastador flexível de fígado na exposição do ângulo esofagogástrico em bypass gástrico em Y-de-Roux (BPGYR) para obesidade mórbida.

\section{MÉTODOS}

Todos os procedimentos foram realizados de acordo com os padrões éticos do Comitê de Pesquisa institucional e/ou nacional, com a declaração de Helsinki de 1964 e suas emendas posteriores. O estudo foi aprovado pelo Comitê de Ética em Pesquisa da Universidade Federal do Ceará sob número 1.482 .503 e pela Comissão Científica do Hospital Geral Dr. César Cals. O consentimento informado foi obtido de todos os participantes incluídos neste estudo (ClinicalTrials. gov Identifier: NCT02926885).

\section{Tipo de estudo}

Trata-se de um estudo prospectivo, monocêntrico, aberto, controlado e comparativo. Cem pacientes obesos foram randomizados em dois grupos diferentes de acordo com o método utilizado para a retração hepática durante a cirurgia.

\section{Participantes de pesquisa}

De abril a agosto de 2016, foram incluídos 100 pacientes obesos de ambos os gêneros, com idade entre 19 e 61 anos, com indicação de cirurgia bariátrica baseada nos critérios da Federação Internacional para a Cirurgia da Obesidade e do Conselho Federal de Medicina (2015).

\section{Técnica cirúrgica}

Todosforam operadosutilizando BPGYR porvia laparoscópica, realizada pela mesma equipe composta por um cirurgião responsável pela execução da técnica, outro atuando como primeiro auxiliar, mais um como segundo auxiliar, um instrumentador e dois anestesiologistas.

Após o tempo pré-operatório, introduziu-se a agulha de Verres, na linha hemiclavicular em hipocôndrio esquerdo rente ao rebordo costal para realização do pneumoperitônio e, posteriormente, a introdução dos trocárteres. Os pacientes foram operados em posição de proclive (aproximadamente $30^{\circ}$ ) e ligeiro (aproximadamente $10^{\circ}$ ) lateral direito para melhor visualização do hipocôndrio esquerdo.

Foram utilizados quatro a seis trocárteres em cada procedimento: um de $12 \mathrm{~mm}$ descartável para introdução do grampeador e quatro a cinco permanentes, sendo um de $10 \mathrm{~mm}$ e 2-4 de $5 \mathrm{~mm}$ (a depender do tipo de afastador utilizado).

A etapa da confecção da bolsa gástrica iniciou-se após a colocação do afastador flexível ${ }^{\circledast}$ de fígado ou do afastador clássico, conforme a randomização. Após a apresentação com boa visualização do ângulo esofagogástrico, na junção esôfago gástrica, introduziu-se a sonda de Fouchet para moldar a bolsa gástrica com grampeamento com cargas azuis. A seguir, isolouse a parte posterior do estômago. Então, uma pequena bolsa gástrica em forma tubular, com $1,5 \mathrm{~cm}$ de diâmetro, $5 \mathrm{~cm}$ de comprimento e capacidade volumétrica aproximada de $60 \mathrm{~cm}^{3}$ foi confeccionada, e, após foi realizada uma sutura de reforço.

Terminado este tempo cirúrgico, fez-se a exclusão de uma alça biliar de $100 \mathrm{~cm}$ a partir do ângulo duodenojejunal. Levou-se este segmento de alçajejunal, antecólica e antegástrica, em direção à bolsa gástrica previamente confeccionada e fixando-a no bordo lateral inferior fazendo-se, então, a gastrojejunoanastomose manual. Posteriormente, mediu-se a alça alimentar, com $120 \mathrm{~cm}$ e confeccionou-se a anastomose jejunojejunal mecânica, utilizando carga branca, seguindo com ofechamento da brecha do grampeador.

Com finalidade de prevenir possíveis hérnias internas, os espaços entre o mesocólon e o mesentério (espaço de Patersen) e o espaço mesentério-mesentério foram fechados. 
Ao final da operação foram aplicados os testes de permeabilidade luminar e o de impermeabilidade das linhas de grampeamento, suturas e anastomoses com azul de metileno. A retirada dos trocárteres foi realizada sob visão direta, para verificar possíveis sangramentos pelos seus orifícios.

\section{Afastador flexível de fígado}

O afastador flexível de fígado ${ }^{\circledR}$ é constituído por um fio agulhado zero de seda de $60 \mathrm{~cm}$ com agulha de 2,5 cm (Ethicon Endo-surgery ${ }^{\circledR}$ ), enluvado por uma sonda de nelaton número 12, cortada em $8 \mathrm{~cm}$ para evitar que o fio traumatizasse o tecido hepático (Figura 1).

\section{Afastador clássico de fígado}

O afastador clássico de fígado é utilizado há vários anos para refração hepática na maioria das gastroplastias realizadas no Brasil. Consiste em uma pinça laparoscópica dentada, autoestática, de $5 \mathrm{~mm}$ de diâmetro, com uma cremalheira em seu cabo (Figura 2).

\section{Grupos do estudo}

O método de afastamento hepático empregado durante as operações dos 100 pacientes foi definido através de uma lista de randomização gerada pelo website http://www.rando1mization.

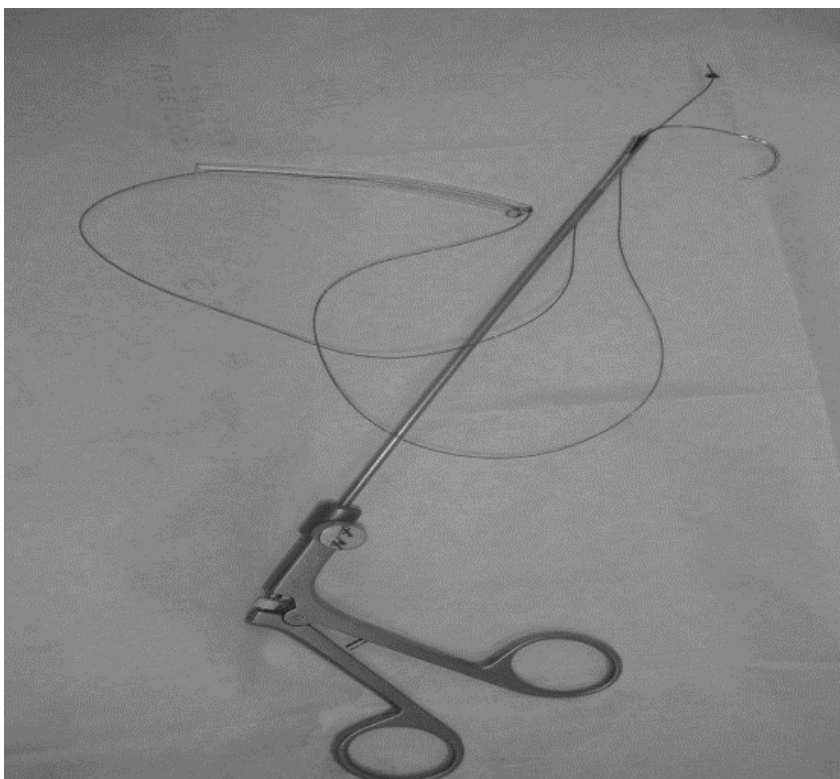

Figura 1 - Afastador flexível de fígado ${ }^{\oplus} \operatorname{composto~por~fio~}$ agulhado zero de seda de $60 \mathrm{~cm}$ enluvado por uma sonda de nelaton número 12 , de $6-8 \mathrm{~cm}$

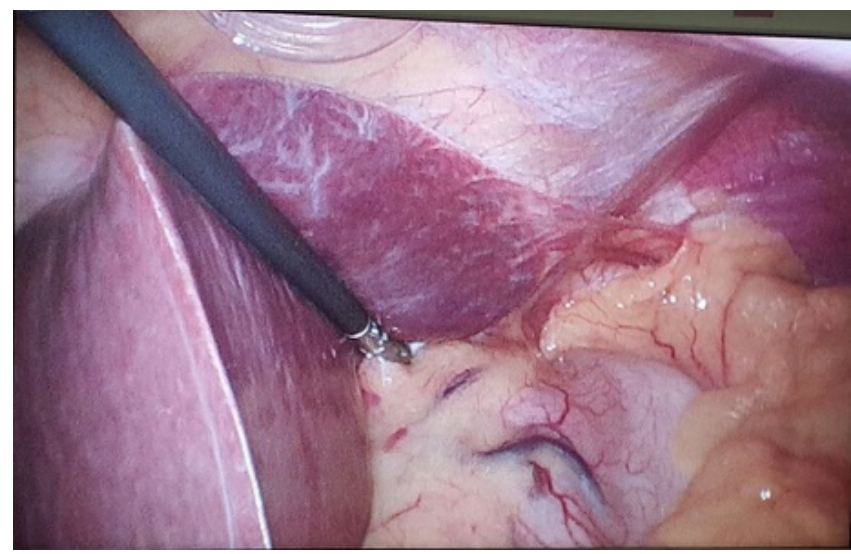

Figura 2 - Afastador clássico de fígado com, na qual cada paciente foi alocado em um dos dois grupos do estudo: controle ou teste.

\section{Grupo controle-Afastador clássico $(n=50)$}

Neste braço do estudo, uma incisão na pele da região subxifoideana foi realizada para introdução de um trocarte de $5 \mathrm{~mm}$. Uma pinça com dentes e cremalheira foi introduzida através deste trocarte, passando entre a face visceral do lobo esquerdo do fígado e o estômago, fixando seus dentes no pilar diafragmático direito. Desta forma, o fígado foi mantido entre a pinça e a parede abdominal, proporcionando rebatimento superomedial do lobo esquerdo do fígado, promovendo a exposição do ângulo esofagogástrico.

\section{Grupo teste - Afastador flexível $(n=50)$}

Neste braço, o afastador flexível de fígado foi introduzido na cavidade abdominal após confecção do pneumoperitônio e aposição dos trocárteres. Com o auxílio da porta-agulhas, a agulha do afastador foi apreendida e o braço direito do pilar diafragmático direito foi transfixado próximo à membrana frenoesofágica. O fio foi tracionado até que a sonda que o reveste serviu de esbarro ao encontrar-se com o pilar. As duas pontas do fio foram, então, tracionadas e a agulha presente em uma das extremidades foi seccionada e retirada da cavidade abdominal. Os dois fios de $5 \mathrm{~mm}$, então apreendidos pela pinça introduzida através do trocarte em epigástrio (trocarte de trabalho da mão esquerda do cirurgião), ligeiramente à direita do paciente, foram tracionados e retirados da cavidade abdominal juntamente com o trocarte em questão que foi prontamente reintroduzido pelo mesmo orifício na pele, por onde doravante, passam os fios e o trocarte.

À medida que foi tracionado, o afastador deslocava o lobo esquerdo do fígado anterior e lateralmente para a direita, provocando exposição ampla da junção esofagogástrica sem traumatizar o tecido hepático e sem a necessidade de incisão suplementar. O sistema foi fixado de forma extracorpórea, por um porta-agulhas de cirurgia laparotômica, que apreendeu os dois fios, rentes à pele.

Em hepatomegalias com esteatose severa, com fígado em tenda, foi realizado afastamento em forma de $\mathrm{V}$. O fio foi fixado através de transfixação do pilar direito e em seguida no diafragma em parede interna anterior mantendo a saída das duas extremidades do fio pelo orifício de trocarte de trabalho da mão esquerda do cirurgião situado em epigástrio.

Ao fim da operação, o afastador foi removido da cavidade abdominal por tração de uma das extremidades do fio e retirado pelo trocarte de trabalho da mão direita do cirurgião (de $12 \mathrm{~mm}$ ).

\section{Variáveis avaliadas}

Para avaliar a eficácia do afastador flexível de fígado, foram avaliados os seguintes parâmetros durante o procedimento cirúrgico.

\section{Desfecho primário}

Visibilidade da junção esofagogástrica: Após a aposição, seja do afastador clássico de fígado, seja do afastador flexível de fígado, foijulgado o grau de visibilidade da junção esofagogástrica, classificada de acordo com a resposta psicométrica (sempre por pelos menos dois cirurgiões em campo cirúrgico) através da escala de Likert ${ }^{2,22}$. O quadrilátero de interesse para o estudo é delimitado pela região que compreende, ou seja, o ligamento hepatogástrico (pars flaccida) e a pequena curvatura gástrica à esquerda, o antro gástrico inferiormente, a grande curvatura gástrica (o fundo gástrico, principalmente) e o baço à direita, e, finalmente, o ângulo esofagogástrico e a junção esofagogástrica superiormente. A ampla visualização de todos estes marcos anatômicos implicou em pontuação 5 na escala (grau de visibilidade ótimo). A medida em que se perdia 
visualização de uma ou mais estruturas, diminuindo o nível de visualização da área de interesse, menor a pontuação: 1 - grau de visibilidade insuficiente; 2 - grau de visibilidade ruim; 3 - grau de visibilidade regular e 4 - grau de visibilidade bom (Figura 3).

\section{Desfechos secundários}

Foram: 1) tempo total de operação, cronometrado do início do pneumoperitônio ao último ponto de sutura na pele; 2) tempo para colocação do afastador clássico de fígado, cronometrado a partir da incisão na pele para introdução do trocarte que possibilitou sua passagem até o afastamento hepático e fixação dos dentes da pinça afastadora ao pilar diafragmático direito; 3) tempo para retirada do afastador clássico de fígado, cronometrado a partir da abertura dos dentes da pinça afastadora até a rafia da pele após sua retirada e do trocarte que permitiu sua passagem; 4) tempo para colocação do afastador flexível de fígado, cronometrado a partir da introdução do afastador na cavidade abdominal até a retirada das duas extremidades dos fios pelo trocarte da mão esquerda do cirurgião e fixação dele externamente com portaagulhas; 5) tempo para retirada do afastador flexível de fígado, cronometrado a partir da abertura do porta-agulhas que o fixa externamente até a retirada do afastador da cavidade abdominal 6) número de incisões na pele para aposição dos trocárteres.

\section{Análise estatística}

As variáveis quantitativas (contínuas e discretas) foram inicialmente analisadas pelo teste de Kolmogorov-Smirnov para verificar a normalidade da distribuição. Para a estatística descritiva, calcularam-se a média e o desvio padrão (dados paramétricos) ou a mediana, intervalo interquartil e valores mínimo e máximo (dados não paramétricos). Comparações entre os grupos de pacientes operados com o afastador clássico e com o afastador flexível foram feitas mediante o uso do teste t para variáveis não

\begin{tabular}{|c|c|c|}
\hline Grau de Visibilidade & Imagem & Pontuação \\
\hline Insuficiente & & 1 \\
\hline Ruim & & \\
\hline Regular & & \\
\hline Bom & & \\
\hline & & \\
\hline & & \\
\hline & & \\
\hline & & \\
\hline
\end{tabular}

Figura 3 - Escala do grau de visibilidade emparelhadas (dados paramétricos) ou do teste de Mann-Whitney (variáveis não paramétricas).Variáveis qualitativas nominais foram expressas como frequência absoluta e relativa e analisadas pelo teste exato de Fisher ou pelo teste de qui-quadrado, conforme o caso. Variáveis qualitativas ordinais, por sua vez, foram expressas como mediana, intervalo interquartil e valores mínimo e máximo e analisadas pelo teste de Mann-Whitney. Nas comparações entre os grupos, determinaram-se a diferença de médias, para variáveis quantitativas, ou de proporções, para variáveis qualitativas, bem como os seus respectivos intervalos de confiança de $95 \%$. Em todas as análises, estabeleceu-se o nível de significância em 0,05 (5\%), sendo considerado como estatisticamente significante um valor $p$ menor que 0,05 . Os softwares IBM SPSS Statistics for Windows ${ }^{\circledR}$ versão 20.0 (IBM Corp., Armonk, NY, USA, 2011) e GraphPad Prism for Windows ${ }^{\circledast}$ versão 5.00 (GraphPad Software, San Diego, California, USA, 2007) foram utilizados para a realização dos procedimentos estatísticos. O último software também foi usado para a elaboração dos gráficos.

\section{RESULTADOS}

\section{Dados demográficos e clínicos}

Cem pacientes obesos foram incluídos $(n=100)$. No grupo do afastador flexível $(n=50), 11$ (22\%) eram homens e 39 (78\%) mulheres. A média de idade dos participantes foi $36,08 \pm 10,77$ anos. No grupo do afastador clássico $(n=50), 23(46 \%)$ eram homens

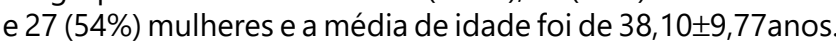
Ogrupo do afastador flexível apresentou diferença estatisticamente significante em relação ao gênero, visto que um número maior de mulheres foi incluído neste grupo quando comparado ao grupo do afastador clássico. Em outras comparações de parâmetros, não houve diferenças significativas entre os braços do estudo. Seus dados demográficos e características clínicas são apresentados na Tabela 1.

\section{Afastador flexível de fígado}

O afastador de fígado flexível disposto em sua forma mais frequente pode ser observado na Figura 4. Os dois fios são tracionados na mesma direção, saindo pelo mesmo orifício do trocarte epigástrico (trocarte de trabalho da mão esquerda do cirurgião) e deslocando o lobo esquerdo anterolateralmente permitindo a adequada visualização do ângulo esofagogástrico.

Para grandes fígados, foi proposto o modelo de afastamento hepático com disposição em V, com o intuito de otimizar a visibilidade do ângulo esofagogástrico (Figura 5).

\section{Tempo cirúrgico}

Não foi constatada diferença estatisticamente significativa entre os dois grupos em relação ao tempo médio de realização das operações $(p=0,748$, Tabela 2$)$.

\section{Colocação e retirada do afastador}

Otempode colocação doafastadorflexível foi significativamente maior do que o afastador clássico $(p<0,001)$. Em contraste, o tempo de retirada do afastador flexível foi significativamente menor do que o afastador clássico $(p<0,001)$.

Quando os tempos de colocação e retirada de ambos os afastadores foram somados, o tempo total para o afastador flexível foi significativamente maior do que para o afastador clássico $(p<0,001)$.

Todos os valores médios, bem como suas comparações estatísticas, podem ser observados na Tabela 2.

\section{Grau de visibilidade da junção esofagogástrica}

A técnica do afastador flexível permite aumentar o grau de visibilidade por meio de uma segunda fixação do fio através de 
Tabela 1 - Dados demográficos e características clínicas dos pacientes operados usando o afastador clássico e flexível

\begin{tabular}{|c|c|c|c|}
\hline \multirow[b]{2}{*}{ Características } & \multicolumn{2}{|c|}{ Grupos - Media (DP) } & \multirow[b]{2}{*}{$\mathbf{p}$} \\
\hline & $\begin{array}{c}\text { Controle } \\
\text { (afastador clássico de fígado) }\end{array}$ & $\begin{array}{c}\text { Teste } \\
\text { (afastador flexível de fígado) }\end{array}$ & \\
\hline$n$ & 50 & 50 & \\
\hline Idade $\left(\right.$ anos) ${ }^{(a)}$ & $38,10 \pm 9,77$ & $36,08 \pm 10,77$ & 0,328 \\
\hline \multicolumn{4}{|l|}{$\operatorname{Sexo}^{(b)}$} \\
\hline Masculino & $23(46,00 \%)$ & $11(22,00 \%)$ & \multirow{2}{*}{0,020} \\
\hline Feminino & $27(54,00 \%)$ & $39(78,00 \%)$ & \\
\hline $\mathrm{IMC}\left(\mathrm{kg} / \mathrm{m}^{2}\right)^{(\mathrm{a})}$ & $41,82 \pm 5,15$ & $40,10 \pm 4,54$ & 0,079 \\
\hline \multicolumn{4}{|l|}{$\mathrm{HAS}^{(b)}$} \\
\hline Presente & $18(36,00 \%)$ & $21(42,00 \%)$ & \multirow{2}{*}{0,682} \\
\hline Ausente & $32(64,00 \%)$ & $29(58,00 \%)$ & \\
\hline \multicolumn{4}{|l|}{ Diabete melito(b) } \\
\hline Presente & $9(18,00 \%)$ & $3(6,00 \%)$ & \multirow{2}{*}{0,12} \\
\hline Ausente & $41(82,00 \%)$ & $47(94,00 \%)$ & \\
\hline \multicolumn{4}{|l|}{ Osteoartropatia ${ }^{(b)}$} \\
\hline Presente & $37(74,00 \%)$ & $41(82,00 \%)$ & \multirow{2}{*}{0,470} \\
\hline Ausente & $13(26,00 \%)$ & $9(18,00 \%)$ & \\
\hline \multicolumn{4}{|l|}{ Dislipidemia $^{(b)}$} \\
\hline Presente & $22(44,00 \%)$ & $23(46,00 \%)$ & \multirow{2}{*}{1,000} \\
\hline Ausente & $28(56,00 \%)$ & $27(54,00 \%)$ & \\
\hline \multicolumn{4}{|l|}{ Apneia do sono(b) } \\
\hline Presente & 25 (51,02\%) & $19(38,00 \%)$ & \multirow{2}{*}{0,228} \\
\hline Ausente & $24(48,98 \%)$ & $31(62,00 \%)$ & \\
\hline \multicolumn{4}{|l|}{ Esteatose hepática(c)14 } \\
\hline 0 (ausente) & $8(16.00 \%)$ & $12(24.00 \%)$ & \multirow{4}{*}{0,177} \\
\hline 1 (leve) & $11(22.00 \%)$ & $18(36.00 \%)$ & \\
\hline 2 (moderada) & $19(38.00 \%)$ & $13(26.00 \%)$ & \\
\hline 3 (grave) & $12(24.00 \%)$ & $7(14.00 \%)$ & \\
\hline \multicolumn{4}{|l|}{$\mathrm{DRGE}^{(\mathrm{c}) 17}$} \\
\hline 0 (ausente) & 28 (56.00\%) & 27 (54.00\%) & \multirow{4}{*}{0,201} \\
\hline 1 (A-Los Angeles) & 18 (36.00\%) & 23 (46.00\%) & \\
\hline 2 (B - Los Angeles) & $3(6.00 \%)$ & 0 (00.00\%) & \\
\hline 3 (C - Los Angeles) & $1(2.00 \%)$ & $0(00.00 \%)$ & \\
\hline
\end{tabular}

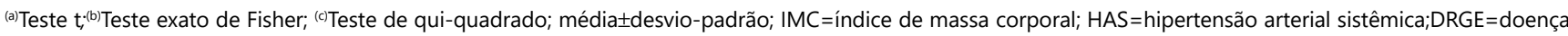
do refluxo gastroesofágico

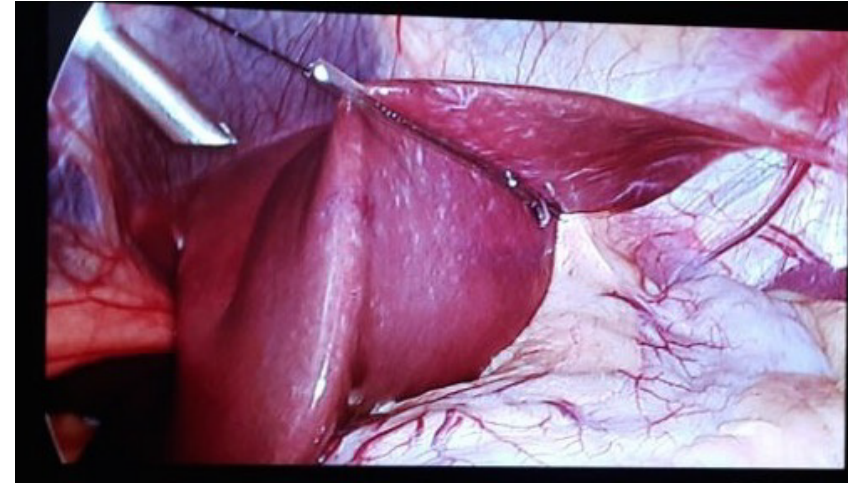

Figura 4 - Afastador de fígado flexível disposto em sua forma mais frequente

transfixação do pilar direito e em seguida no diafragma em parede interna anterior. Dessa forma, avaliou-se o grau de visibilidade nestes dois momentos. Observou-se que o grau do afastador flexível, quando fixado em apenas dois pontos (no primeiro momento de colocação), era significativamente menor do que $o$ afastador clássico forneceu $(p=0,003)$.Entretanto, quando o afastador flexível de fígado foi fixado em três pontos "em V" (segundo momento), não foi observada diferença estatisticamente significante entre o nível de visibilidade fornecido pelo afastador clássico e flexível $(p=0,743)$.

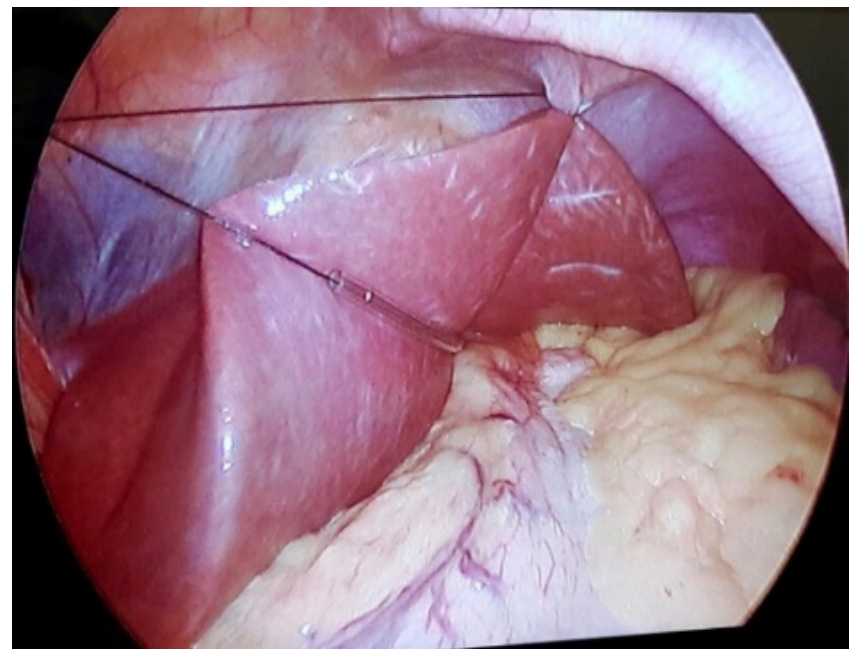

Figura 5 - Modelo de afastamento hepático proposto para grandes fígados

Ainda, quando avaliada somente o primeiro momento de colocação do afastador flexível, a proporção de pacientes nos quais a visibilidade da junção esofagogástrica foi graduada como ótima ou completa foi significantemente menor (74\%, $p=0,012$ ) do que entre os sujeitos operados com o afastador clássico (94\%). Enquanto, quando considerado o segundo 
Tabela 2 - Valores médios e comparação estatística do tempo cirúrgico, tempo de colocação e retirada e tempo total de colocação e retirada do afastador, observados nos pacientes operados com afastador clássico e flexível

\begin{tabular}{|c|c|c|c|c|c|}
\hline \multirow[b]{2}{*}{ Tempo } & \multicolumn{2}{|c|}{ Grupos - Média (DP) } & \multicolumn{3}{|c|}{ Diferença } \\
\hline & $\begin{array}{c}\text { Controle } \\
\text { (Afastador clássico de fígado) }\end{array}$ & $\begin{array}{c}\text { Teste } \\
\text { (Afastador flexível de fígado) }\end{array}$ & $\mathbf{p}$ & $\begin{array}{l}\text { entre as } \\
\text { médias }\end{array}$ & $95 \% \mathrm{Cl}$ \\
\hline Cirurgia(minutos) & $85,70 \pm 14,06$ & $86,66 \pm 15,66$ & $p=0,748$ & $-0,96$ & $-6,88$ a 4,96 \\
\hline Colocação(segundos) & $25,64 \pm 18,62$ & $120,96 \pm 44,45$ & $p<0,001$ & $-95,32$ & $-108,87$ a $-81,77$ \\
\hline Retirada(segundos) & $33,18 \pm 11,35$ & $10,90 \pm 9,54$ & $p<0,001$ & 22,28 & 18,11 a 26,45 \\
\hline $\begin{array}{l}\text { Colocaçãoe retirada } \\
\text { (segundos) }\end{array}$ & $58,82 \pm 23,53$ & $131,86 \pm 48,45$ & $p<0,001$ & $-73,04$ & $-88,18$ a $-57,90$ \\
\hline
\end{tabular}

$\mathrm{DP}=$ desvio padrão; $95 \% \mathrm{IC}=$ intervalo de confiança de $95 \%$ da diferença entre as médias; *Dados correspondentes àanálise dos 50 pacientes de cada grupo

momento, quando este se fez necessário, todos os pacientes do grupo afastador flexível obtiveram visibilidade ótima ou completa (grau de visualização 1 ou 2), porém sem diferença estatística significante com relação ao grupo clássico.

Número de incisões na pele para colocação de trocárteres

Na comparação estatística do número de incisões na pele para colocação do trocarte e o número observado em pacientes operados com afastador flexível (média de cinco incisões) foi significativamente menor do que em pacientes operados com afastador clássico (média de seis incisões, $p<0,001$ ).

\section{DISCUSSÃO}

\section{Afastadores hepáticos}

O BPGYR é a intervenção de referência do tratamento cirúrgico da obesidade mórbida e a adequada exposição e visualização da junção esofagogástrica são fundamentais para sua realização ${ }^{5}$. 0 efetivo afastamento hepático deve permitir acesso fácil, visualização adequada do campo operatório, espaço para que as manobras sejam realizadas com segurança, traumatizando minimamente os tecidos e planos anatômicos com a maior preservação e menor manipulação possível dessa região.

A fim de resolver as questões acima, muitos cirurgiões desenvolveram técnicas projetadas para a retração do fígado $0^{4,7,8,15,16,19}$. No entanto, embora existam vários estudos descrevendo diferentes tipos de afastadores hepáticos, poucos são os comparando métodos.

O afastador de Nathanson, a fita de suspensão hepática e o método "V-LIST" foram comparados em um estudo randomizado com 60 pacientes. Como resultado, o tempo necessário para a aposição do retrator V-LIST foi consideravelmente maior do que para o retrator de Nathanson. No entanto, os autores consideraram que a maior familiaridade com o esse afastador pode ter interferido no resultado. Invariavelmente, para o afastador de Nathanson, uma incisão adicional na pele era necessária, limitando seu uso para procedimentos de portal único. Além disso, o aumento dos níveis séricos de enzimas hepáticas foi significativamente maior com esse afastador, bem como mais dor pós-operatória foi observada quando comparado aos outros dois métodos ${ }^{6}$.

Em outro estudo recente, dois afastadores de fígado padrão, Nathanson e PretzelFlex, foram comparados usando dados retrospectivos de 167 pacientes (93 no grupo de afastadores de Nathansone 74 no afastador de PretzelFlex) submetidos a BPGYR. Os autores observaram duração semelhante da operação em ambos os grupos. Os pacientes do grupo afastador de Nathanson apresentaram níveis mais elevados de alanina transaminase e proteína $C$ reativa. $O$ dano hepático foi significativamente menor no grupo do afastador PretzelFlex (que por sua vez está associado a menos dor pós-operatória e náusea) quando comparado ao afastador de Nathanson ${ }^{9}$.

\section{Afastador flexível vs.afastador clássico - aspectos relevantes}

Após a conclusão deste estudo, todas as operações foram concluídas com um nível de visibilidade adequado da junção esofagogástrica, sugerindo a não inferioridade do afastador flexível do fígado quando comparado ao afastador clássico do fígado. Todavia, os seguintes aspectos merecem considerações:o afastador flexível, por exigir mais manobras (inclusive com manipulação da agulha), demandou mais tempo para sua colocação, levando à diferença estatisticamente significante quando comparado ao afastador clássico, que possui manuseio mais simples durante sua colocação; para retirada, entretanto, houve diferença a favor do afastador flexível. Enquanto este só precisa ser puxado para fora da cavidade abdominal após a abertura do porta-agulha que o sustenta na parte externa, a remoção do afastador clássico só é concluída após a sutura da pele incisada. Por fim, o tempo cirúrgico total foi semelhante para os dois afastadores.

\section{Aspectos positivos do afastador flexível}

Apesar da diferença de tempo, na comparação do tempo operatório total, parâmetro cirúrgico de real interesse, não foi observada relevância estatística a favor de nenhum dos afastadores, o que pode indicar indiretamente a adequada visualização oferecida pelo afastador flexível. Invariavelmente, sempre foi necessário carregar um trocarte a mais na utilização do afastador clássico, pois para sua aposição é necessário um orifício próprio na pele.

Em tempos de cirurgias minimamente invasivas, quando se busca o mesmo ou o melhor resultado, porém causando o menor dano possível, seja relacionado a resposta inflamatória ao trauma, seja a possibilidade de menor sangramento, de hérnias incisionais, de infecção ou de cicatrização hipertrófica que cada nova incisão pode representar, essa é uma indubitável vantagem da utilização do afastador flexível de fígado.

Outro ponto a ser considerado, embora não tenha sido demonstrado aqui, é a injúria hepática. Ela pode ser minimizada pelo uso de um afastador flexível, pois este se molda à superfície do fígado, diminuindo a chance de fraturá-lo do que afastadores rígidos, como aqueles utilizados neste estudo como controle. Importante também é a característica espacial do afastador flexível, que, sendo totalmente intracavitário, não é suscetível a colisões com a pinça ou com os braços de cirurgiões ou auxiliares. Essa característica pode se tornar ainda mais importante se forem consideradas operações realizadas com assistência robótica, nas quais os braços do robô podem colidir com o paciente ou com uma haste de afastador que se projeta para fora do paciente, predispondo acidentes.As incisões do trocarte podem ser focos de infecção, sangramento, dor, cicatrizes hipertróficas ou hérnias de parede que podem complicar com obstrução intestinal, que, embora raras, são descritas mesmo com punções de $5 \mathrm{~mm}$. Assim, durante os procedimentos laparoscópicos, são utilizados trocárteres por onde passam os instrumentais e, na medida do possível, utiliza-se o menor número deles, desde que a segurança do ato cirúrgico não seja comprometida. Porém, mesmo com a necessidade de uma 
incisão a menos nos pacientes do grupo do afastador flexível, não foi observada mudança de rotina pós-operatória quando comparado ao grupo do afastador clássico.

Uma vez colocado, o afastador clássico deixa poucas alternativas de reposicionamento para otimizar o nível de visibilidade da junção esofagogástrica. Se inadequada a ponto de interferir com a segurança do procedimento, somente pode ser corrigida com a introdução de uma nova pinça afastadora por um porte adicional de trocarte. Além disso, como na prática clínica, ao ocupar uma das mãos do cirurgião auxiliar durante o tempo principal da operação para manter a retração hepática adequada, evitará o uso de um trocarte auxiliar do tamanho, o que minimizaria ainda mais o número de incisões na parede. No entanto, o auxiliar trabalharia com apenas uma mão.

Ao evitar-se uma punção a mais, a capacidade de ajuda do auxiliar fica limitada. Por outro lado, o afastador flexível permite o posicionamento variável de uma de suas alças (um de seus fios constituintes), o que proporciona versatilidade para uso em fígados de diferentes formatos.

Frente aos resultados obtidos, observou-se que o afastador flexível apresenta as condições técnicas para ser validado como instrumento de retração hepática para exposição adequada e segura da junção esofagogástrica em cirurgias bariátricas e em outros procedimentos de abdome superior em que o fígado dificulta a visibilidade. Além disso, por permitir a exposição da parte superior do abdome sem a necessidade de incisões para isto, esse afastador atende às características de um equipamento cirúrgico minimamente invasivo e pode ser utilizado em operações de portal único. Ademais, por não exigir mecanismos de fixação externos, pode ser uma alternativa aos afastadores usados atualmente em cirurgia robótica, devido ao risco mínimo de colisão com os braços do robô.

Este estudo apresenta algumas limitações, principalmente no que se refere ao registro das informações do processo cirúrgico, como quantidade de medicamentos necessários para analgesia, registro das complicações durante e após a operação, avaliação da dor na recuperação e tempo de internação. Outro grupo controle também poderia ter sido utilizado empregando outro afastador de fígado, como o de Nathanson, por exemplo.

\section{CONCLUSÃO}

O afastador flexível de fígado mostrou-se seguro, eficaz, ergonômico, barato e com perfil estético satisfatório. Além disso, não foi necessária a utilização de instrumentos específicos ou curva de adaptação e treinamento, tornando-o adequado para ser utilizado como alternativa aos afastadores atualmente disponíveis.

\section{REFERÊNCIAS}

1. AhmadA,Arellano JJ, AgarwalaA,AhmadZ,AhmadZ.A percutaneous technique of liver retraction in laparoscopic bariatric \& upper abdominal surgery. Surg Obes Relat Dis. 2016;12(8):1626-1629. doi.10.1016/j.soard.2016.08.014.

2. Allen, I. and Seaman, C., 2007. Statistics Roundtable: Likert Scales And Data Analyses. [online] Available at: <http://asq.org/qualityprogress/2007/07/statistics/likert-scales-and-data-analyses.html> [Accessed 11 November 2017].

3. Batista Marchesini J. A Safer and Simpler Technique for the Duodenal Switch: To the Editor: Obes Surg. 2007;17(8):1136. doi. 10.1007/s11695-007-9192-1.

4. GalvaniCA,ChohM, GorodnerMV.Single-incisionsleevegastrectomy using a novel technique for liver retraction. JSLS: 2010;14(2):22833.doi.10.4293/108680810X12785289144278.
5. Garrido Júnior AB, Elias AA, Oliveira MR, Ito RM, Shirozaki HY. Histórico do tratamento cirúrgico da obesidade. In: Tratado de obesidade [2ed.] 2015. Ed.Guanabara, São Paulo.

6. Goel R, Shabbir A, Tai CM, Eng A, Lin HY, Lee SL, Huang CK. Randomized controlled trial comparing three methods of liver retraction in laparoscopic Roux-en-Y gastric bypass. Surg Endosc. 2013;27(2):679-84. doi. 10.1007/s00464-012-2438-6.

7. Hamzaoglul,KarahasanogluT,AytacE, Karatas A, BacaB. Transumbilical totally laparoscopic single-port Nissen fundoplication: a new method of liver retraction: the Istanbul technique. J Gastrointest Surg. 2010;14(6):1035-9. doi. 10.1007/s11605-010-1183-1.

8. Huang CK, Houng JY, Chiang CJ, Chen YS, Lee PH. Single incision transumbilicallaparoscopic Roux-en-Ygastric bypass: afirstcasereport. Obes Surg. 2009;19(12):1711-5.doi: 10.1007/s11695-009-9900-0.

9. Midya S, Ramus J, Hakim A, Jones G, Sampson M. Comparison of Two Types of Liver Retractors in Laparoscopic Roux-en-Y Gastric Bypass for Morbid Obesity. Obes Surg. 2020;30(1):233-7. doi. 10.1007/s11695-019-04142-6.

10. Moura Jr LG, Feitosa HC, Machado FHF, Babadopulos RF, Feijó FC, Fernandes SD. Minimizing portals with liver malleable retractor in bariatric laparoscopic acess, N.O.T.E.S. robotic. P.21 - Poster Sessions - 5th Congress of the International Federation for the Surgery of Obesity and Metabolic Disorders European Chapter (IFSO-EC) Barcelona April, 26-28, 2012.

11. Moura-Júnior LG, Castro-Filho HF, Machado FH, Babadopulos RF, Feijó FD, Fernandes SD. Ports minimization with mini-port and liver flexible retractor: an ergonomic and aesthetic alternative for single port in laparoscopic gastric bypass. ABCD. Arq Bras Cir Dig. 2014;27 Suppl 1(Suppl 1):77-9. doi: 10.1590/s0102-6720201400s100019.

12. Nguyen NT, Longoria M, Gelfand DV, Sabio A, Wilson SE. Staged laparoscopic Roux-en-Y: a novel two-stage bariatric operation as an alternative in the super-obese with massively enlarged liver. Obes Surg. 2005;15(7):1077-81. doi. 10.1381/0960892054621062.

13. Pasenau J, Mamazza J, Schlachta CM, Seshadri PA, Poulin EC. Liver hematoma after laparoscopic nissen fundoplication: a case report and review of retraction injuries. Surg Laparosc Endosc Percutan Tech.2000;10(3):178-81.doi.10.1097/00019509-200006000-00016.

14. Saadeh S, Younossi ZM, Remer EM, Gramlich T, Ong JP, Hurley M, Mullen KD, Cooper JN, Sheridan MJ. The utility of radiological imaging in nonalcoholic fatty liver disease. Gastroenterology. 2002;123(3):745-50. doi.10.1053/gast.2002.35354.

15. Saber AA, El-Ghazaly TH. Early experience with single incision transumbilical laparoscopic adjustable gastric banding using the SILS Port. Int JSurg.2009;7(5):456-9. doi. 10.1016/j.jijsu.2009.07.004.

16. Sakaguchi $Y$, Ikeda O, Toh $Y$, Aoki Y, Harimoto N, Taomoto J, Masuda T, Ohga T, Adachi E, Okamura T. New technique for the retraction of the liver in laparoscopic gastrectomy. Surg Endosc. 2008;22(11):2532-4. doi. 10.1007/s00464-008-9801-7.

17. SamiSS, RagunathK. TheLosAngelesclassification of gastroesophageal reflux disease. Video journal and Encyclopedia of GI Endoscopy. 2013;1(1):103-4. doi.org/10.1016/S2212-0971(13)70046-3.

18. Santoro $S$, Aquino CGG, Mota FC, Artoni RF. Does evolutionary biology help the understanding of metabolic surgery? A focused review. Arq Bras Cir Dig. 2020;33(1):e1503. doi: 10.1590/0102$672020190001 \mathrm{e} 1503$

19. Shibao K, Higure A, Yamaguchi K. Disk suspension method: a novel and safe technique for the retraction of the liver during laparoscopic surgery (with video). Surg Endosc. 2011;25(8):2733-7. doi.10.1007/s00464-011-1614-4.

20. Stein J, Stier C, Raab H, Weiner R. Review article: The nutritional and pharmacological consequences of obesity surgery. Aliment Pharmacol Ther. 2014;40(6):582-609. doi: 10.1111/apt.12872.

21. Stoll A, Rosin L, Dias MF, Marquiotti B, Gugelmin G, Stoll GF. Early Postoperativecomplications in Roux-en-Y gastric bypass. Arq Bras Cir Dig.2016;29(Suppl 1):72-74. doi:10.1590/0102-6720201600S10018.

22. SurveyMonkey. 2017. Likert Scale: What It Is \& How To Use It | Surveymonkey. [online] Available at: < https://www.surveymonkey. com/mp/likert-scale/> [Accessed 10 November 2017]. 